\title{
Creación artística: el artista y la comunidad*
}

\author{
Artistic creation, links between artist and community
}

\section{Resumen}

La intención de este texto es reconocer diversos escenarios, épocas y obras artísticas específicas que permiten una aproximación a distintas maneras en que algunos artistas plásticos y visuales se desligaron de los procesos creativos individuales convencionales y eligieron actos artísticos colectivos. Se orienta a señalar el sentido de ruptura y desobediencia que asumen los artistas en sistemas culturales tradicionales, lo cual, lleva a identificar estrategias empleadas para generar experiencias estéticas, desarrollar conocimiento y legitimar obras mediante procesos creativos participativos, que permitan, seguir algunas premisas históricas globales y locales con el fin de analizar actos creativos que surgen entre el artista y la comunidad necesarios para el desarrollo del proyecto de investigación titulado Prácticas y saberes comunitarios para la creación artística. "Aportes a la construcción de paz en el suroccidente colombiano". Esta propuesta plantea en su primera fase la identificación, análisis y valoración de diferentes prácticas artísticas comunitarias que sirven como insumo para desarrollar procesos creativos que articulan saberes y acciones entre el colectivo de mujeres Ashampa Awá en San Juan de Pasto, Departamento de Nariño y los investigadores del programa Licenciatura en Educación de la Fundación Universitaria Católica Lumen Gentium. En las páginas que siguen se propone un relato reflexivo que aborda la noción de creación artística como estado de exploración, proceso de conocimiento e interacción entre el artista plástico-visual y las prácticas comunitarias.

Palabras clave: creación, artista, comunidad, participación, contexto social.

* Artículo producto de investigación Pyscca Prácticas y saberes comunitarios para la creación artística. "Aportes a la construcción de paz en el suroccidente colombiano" programa Licenciatura en Educación Artística de UNICATólicA.

** Licenciada y magíster en Artes Visuales de la Universidad del Valle y La Universidad Nacional Autónoma de México, docente investigadora para la Fundación Universitaria Católica Lumen Gentium, Cali, Colombia. Miembro del grupo de investigación Educarte. Correo electrónico: patafur@unicatólica.edu.co. Orcid: https://orcid.org/0000-0001-7413-0607. 
Sumario: 1. Creación artística; 2. El artista fuera del taller; 3. Prácticas artísticas en América Latina; 4. Experiencias artísticas desde las artes plásticas en Colombia.

\section{Abstract}

The intention of this text is to recognize various scenarios, epochs, geographies and specific artistic works, which allow an approach to various ways in which some visual and plastic artists separated themselves from conventional individual creative processes and chose collective artistic acts. It aims at pointing out the sense of rupture and disobedience assumed by artists in traditional cultural systems, and, which entails identifying strategies used to generate aesthetic experiences, develop knowledge and legitimize works through creative participatory processes, which allows following some historical premises to try to answer: how can we analyze the creative acts that arise collectively between artist and community?, a concern that arises in the framework of the research project entitled: Community practices and knowledge for artistic creation. "Contributions to the construction of peace in the southwest of Colombia". In its first phase it proposes the identification, analysis and assessment of different community artistic practices that serve as input to develop creative processes that articulate knowledge and practices of the Ashampa Awá women's group in San Juan de Pasto, Nariño Department, and researchers of the pregraduate Education program of the Fundación Universitaria Católica Lumen Gentium.. The pages that follow offer a reflexive story that addresses the notion of plastic-visual artistic creation as a state of exploration, process of knowledge and interaction between the artist and community practices.

Keywords: creation artist, community, participation, social context.

Summary: 1. Artistic creation; 2. The artist outside the workshop; 3. Artistisc practices in Latin
America; 4. Artistic experience from the plastic arts in Colombia.

\section{Creación Artística}

Es importante iniciar con una definición sobre lo que se entiende por creación artística. El término hace referencia a la capacidad del ser humano de expresar sus ideas y sensaciones por medio de la música, danza, literatura, plástica, teatro, cine y otras manifestaciones audiovisuales y digitales que evidencian una experiencia sensible. La creación artística es el acto que da a luz toda producción de sentido que constituye todo lenguaje que interviene en la comunicación humana (Mendogo, 2004, p. 808). Esta capacidad es, sin duda, una práctica esencial en la vida del ser humano que da cuenta de una época, cultura, grupo social e individuos que dan forma a sus ideas e intuiciones. Para Herbert Read los dotes intuitivos pertenecen al artista, es así que plantea que

[...] para expresar su intuición usará el artista la materia que las circunstancias y el tiempo ponen en sus manos: en una época arañará los muros de su cueva, en otra construirá o decorará un templo o una catedral, en otra pintará sobre tela para un círculo limitado de connoisseurs (Read,1954, p. 147)

Aunque el autor se refiere a la contribución plástica, en general, los artistas han ocupado un rol importante en la sociedad, ya que el lenguaje artístico, bien sea de tipo plástico, musical, literario, entre otros elementos de expresión, tiene un valor estético que permite experimentar una especie de libertad por medio de la forma, el color, la línea, el espacio; y al mismo tiempo consigue comunicar y producir intercambios culturales que logran construir mundos posibles y muchas veces problematizar la cotidianidad, con lo cual cobra importancia según el periodo histórico al que pertenece.

Así mismo, esta capacidad está influenciada por la relación economía-cultura, dos temas distintos 
pero que forman una alianza innegable. $Y$ es que, desde el siglo XIX y en la transición de un sistema económico feudal a una organización de la economía capitalista, este binomio se prioriza en países que son potencia mundial y la condición de artista amparado por mecenas se desdibuja, lo cual provoca que él mismo se desvincule de ambientes tradicionales como museos, galerías y espacios fuera de la disertación hegemónica, y extiende su interés en el mercado para consolidar su estilo y a la par, aumentar su atención a problemas socio-culturales -propios y ajenos-, halla otros modos de producir arte y exhibirlo en sociedad.

Es el caso de algunos pintores franceses de finales de aquel siglo, quienes retomaron una práctica centenaria: pintar al aire libre, reacción que obedeció en parte a la aparición de la fotografía y su utilidad para capturar fielmente la realidad, capacidad que, hasta ese momento se le otorgaba al pintor con su virtud casi divina del manejo del dibujo y el color. También porque el realismo de aquella época promovido por la Academia de Bellas Artes tuvo poca acogida entre los impresionistas1, movimiento conformado por pintores que "eclosiona en todo su esplendor durante un momento históricamente muy complicado, marcado por la guerra franco-prusiana y los sucesos de la comuna, que convulsionan París" (Fundación Mapfree, 2010, p. 3).

Para Édouard Manet (1832-1883), Edgar Degas (1834-1917), Claude Monet (1840-1926), Berthe Morisot (1841-1895), Camille Pisarro (1830-1903), Auguste Renoir (1841-1919), Alfred Sisley (1839-1899), entre otros artistas, el paisaje y la vida cotidiana tenía relevancia a tal grado que debía en cada instante ser capturada con pinceladas dinámicas, de tonos puros y figuras ligeras. Esta nueva actitud provocó escándalos transitorios en el marco del salón de rechazados (Salon des Refusé, 1863), o La sociedad de los artistas independientes (La Société des Artistes
Indépendants, 1884), exhibiciones creadas para desafiar la estructura gremial, académica y tradicional del Salón de Paris, evento desarrollado desde el siglo XVII en aquella ciudad, considerada, en ese momento, la capital mundial del arte y el lugar donde el Estado compraba arte e indudablemente, el artista alcanzaba su valía. Estos sucesos imprimieron nuevos valores en un intento de ajustarse al mundo moderno, que en definitiva, generó aportes que cambiaron la manera hacer y entender la pintura.

\section{El artista fuera del taller}

Hacia el siglo XX el estar fuera del taller obedeció al interés por priorizar sensaciones e instantes colmados de un nuevo espíritu en medio de una época agitada. El surgimiento de las vanguardias históricas no solamente evidenció la necesidad de transformar la función del arte, también la urgencia de cambios sociales, políticos e ideológicos que demandaba el inicio de la centuria ,atestada de tensiones políticas y conflictos económicos entre países soberanamente industrializados; Reino Unido, Francia, Alemania, Rusia y Austria enfrentados para proteger su comercio y conquistar otros mercados, por lo que creaban políticas agresivas y hegemónicas que desataron la Primera Guerra Mundial.

Es así que la participación de los artistas en los postulados de los países en guerra fue ineludible, la sociedad capitalista y los estados socialistas exigieron su intervención directa, ya que el subsidio que recibían los obligaba a cumplir su deber patriótico o a vivir en el exilio, esto ocasionó sucesos infaustos en medio de tantos cadáveres y ruinas. La inconformidad se manifestó de forma colectiva, la transformación plástica se abrió camino, materiales como hule, cartón, trozos de madera, periódico usado, vidrio y nuevos metales cobraron protagonismo y se introdujeron en la pintura y la escultura, lo cual les otorgó un carácter novedoso. Las experimentaciones

1 El término impresionismo origina de la expresión que utiliza el crítico de arte Lovis Leroy en un artículo publicado en 1974 tras recorrer la exposición organizada en el taller de Nadar. Leroy calificó de forma peyorativa a la obra "Impresión: sol naciente" de Claude Monet y desde ese momento los artistas involucrados no dudaron en adoptar este nombre. 
plásticas motivaron el uso de técnicas como collage, gratagge, frottage y fotomontaje, incluyendo experiencias visuales que involucraron la fotografía y el cine, así mismo, la revisión sobre distintas culturas y la conjugación con otras disciplinas como literatura, teatro, danza, arquitectura y música se hicieron presentes. "Cuánta energía histórica que, antes que, en la violencia social y política, encontró su liberación en el arte de las vanguardias" (Báez, 2000, p. 239). Fueron ismos que dieron apertura a formas que ya no tienen reglas fijas de representación, sino múltiples posibilidades de crear y exhibir. Por tanto, algunas expresiones literarias, plásticas, escenográficas y sonoras irrumpen en lo habitual y se arrojan a las calles como estrategia de ataque a la acostumbrada actitud contemplativa del espectador, custodiada por el arte oficial y sus paredes blancas que cautivan los sentidos.

En el lenguaje plástico, una obra puntual que cabe nombrar en este escenario es Merzbau, del artista Kurt Schwitters, un proyecto creado con objetos cotidianos que formó una especie de columna infinita incrustada al interior de la casa del artista. "Una especie de collage abstracto y compuesto de grutas, columnas y objetos encontrados, siempre cambiantes y en constante expansión" (Thomas, 2012, p. 1).

Este proceso creativo, marcó de manera intencional la diferencia del objeto con relación al espacio y aunque nunca se pudo terminar a causa de la persecución nazi y los bombardeos de la Segunda Guerra Mundial -que obligó al artista a trasladarse continuamente hasta su muerte en 1948-, los historiadores narran la coherencia de esta pieza con respecto a uno de los ideales de vanguardia: fusionar arte y vida. Al respecto, el mismo Schwitters indicó en un texto escrito en 1920 para la revista publicada en Múnich, Alemania, de 1918 a 1921, Der Ararat: Glossen, Skizzen und Notizen zur neven Kunst (El ararat: glosas, bocetos y notas sobre arte nuevo): "se puede estar alegre incluso entre la basura y eso es lo que hice: la recogí, la encolé, la clavé, y la llamé
Merz" (Schwitters, 1995). Y justamente Merzbau es una referencia importante convertida en hito, porque posteriormente, influyó en los artistas que además de pinturas y esculturas, crearon instalaciones y acciones, diversificaron así las formas y lugares de producción artística y otorgaron legitimidad a los actos creativos que se desarrollaban fuera de museos e instituciones artísticas oficiales.

No cabe duda de que los artistas accionan y las instituciones reaccionan, el Museo de Arte Moderno (MoMa) se fundó en el continente americano en 1929 y de la mano del director Alfred H. Barr, se rejuveneció el concepto de museo, dispuesto a proteger, promover y difundir las diversas producciones artísticas internacionales, incluso aquellas obras vanguardistas de difícil aceptación en su momento. A la par, gradualmente se extiende el campo de acción al incorporar arquitectura, diseño, fotografía y cine, lo cual convierte el concepto de museo-almacén en museo-laboratorio organizado, vivo y didáctico. Así, Fernando Huici (1981) realiza una afirmación acerca del fortalecimiento de la plástica moderna impulsada por Barr :

[...] Es sin duda, 1936 el año crucial en la carrera expresiva de Barr con las gigantescas exposiciones tituladas: Cubismo, arte abstracto y arte fantástico, dadá y surrealismo, las cuales ofrecerían al público y a los artistas neoyorquinos un panorama exhaustivo de los movimientos que habían de sentar las bases de la gran generación pictórica de posguerra norteamericana. (p. 13)

De acuerdo con lo anterior, en la década de 1940 se forjó la idea de un arte estadounidense que confortó a la sociedad y dominó la escena artística mundial. Esta atribución hizo posible -en gran medida- que el foco del arte moderno saliera de Paris y se alojara en Nueva York, lo cual resultó en el expresionismo abstracto, un movimiento que destaca como figura principal a Jackson Pollock (1947-1948), quien 
describe su quehacer para la revista de Vanguardia Possibilities de la siguiente manera: "cuando estoy dentro de mi pintura no estoy consciente de lo que estoy haciendo. Solo después de un periodo de "acostumbrarme" veo lo que he hecho en realidad" ( p. 48 ). Esta forma de producción que invalidó la idea de crear un lenguaje al servicio de la sociedad se consiguió implantar y tener éxito decisivamente en plena Guerra Fría, que inicia una vez acaban I y II Guerra Mundial. De esta manera, logra presentar a nivel mundial un arte norteamericano genuino, emancipado de Europa y nuevo modelo de la cultura occidental en el que algunos artistas representativos estaban totalmente despolitizados. "El artista intersubjetivo inventa, a partir de la experiencia personal, crea desde un mundo interno, en vez de externo" (Guilbaut, 2007, p. 227).

\section{Prácticas artísticas en américa latina}

Algo distinto ocurrió en América Latina, ya que los acontecimientos históricos y culturales determinaron el desplazamiento de la acción artística hacia el terreno político que se entrelaza con el campo social. Hechos como el surgimiento de la Revolución Cubana, la lucha que libraron Vietnam y África por su libertad contra países poderosos, movimientos sociales liderados por sacerdotes que articularon el Marxismo y el cristianismo, fueron todos eventos coyunturales, que promovieron un verdadero cambio en beneficio de los más oprimidos e involucraron un giro de pensamiento y de forma en las artes.

Para la década de 1968 los grupos estudiantiles, obreros e intelectuales se enfrentaron a las directrices represivas del Estado, los artistas comprometidos decidieron unirse a esta lucha popular y buscar espacios mucho más democráticos, se apartaron del mundo oficial. En el programa de Tv Mestizo. 12 militancia artística, Graciela Carnevale artista plástica argentina que participó en la realización colectiva artístico-política Tucumán Arde, rememora:
[...] Salirse de las Galerías, alquilar un local en una galería comercial y de esa manera autogestionarnos, armar nuestras propias muestras, nuestros montajes, hacer la publicidad, escribir nuestros textos, eso nos pone en una situación de autonomía frente a todo el sistema y en la búsqueda también de un nuevo público. (2008)

La dirección que tomó el lenguaje artístico frente a la crítica institucional se agudizó. El ambiente hostil y la censura se intensificaron cuando América Latina se vió sacudida por las dictaduras y las guerras civiles de cada nación. La violencia estableció la fuerza que hizo volcar la creación sobre el quehacer político en aras de un mundo mejor. La realidad debía ser denunciada, comunicada, señalada; el arte se convirtió en información, un medio para visibilizar los abusos del Estado contra la población civil, las masacres silenciadas, las desapariciones y la represión de todo tipo. Los lenguajes artísticos tomaron nuevas formas, desde distintas geografías, por colectivos o individuos permeados y conectados por condiciones de vida sobrellevadas en un escenario cruel, autoritario y sangriento.

El desafío del lenguaje pictórico involucró nuevas formas artísticas, happenings, ambientaciones, performances, creó los cimientos para una crítica política e ideológica al sistema del arte. la irrupción de la acción en el campo de las artes plásticas, a partir de 1960, consiguió alterar la idea de una historia lineal y única, expresó múltiples historias fracturadas que le permitieron a la experiencia creativa ocupar un lugar comprendido, necesariamente, en la temporalidad, y permitir al artista integrarse más con su entorno. La historiadora de arte Andrea Giünta (2014) precisa:

[...] Cuando irrumpen los Parangolés de Oiticica o las terapias con objetos relacionales de Lygia, objetos para poner en actividad nuevas zonas mínimas de la percepción, 
realizados con materiales simples (bolsas de plástico, agua, piedras y arena) y activados desde el cuerpo, estamos en un momento distinto. Incluso caminando la cinta de Moebius, en el papel de Clark, que el participante corta por el medio hasta no poder avanzar más -una obra que es lo que dura, el tiempo en el que se recorre el papel, dividiéndolo siempre en dos- da cuenta de una diferencia radical respecto de sus trabajos anteriores [...] estas piezas de Hélio y Lygia, involucran elementos completamente distintos. Procesos, tiempo, movimiento, cuerpos. (pp. 15-16)

Así, el gesto inquieto cobra valor, más allá del bastidor, la roca esculpida y la materialidad del objeto. El cuerpo, en tiempo-espacio real, logra indagar otros cuerpos a través de los sentidos, suscita la participación y constata que el acto, tan fugaz como eficaz, consigue desvanecer las fronteras entre el público y la obra. De tal manera que la acción de intervenir en los espacios urbanos, naturales o privados, exige a quien la ejecuta, prescindir de los espacios oficiales.

Consecuencia de la oleada de violencia y la acción de resistencia, emergieron en América Latina durante 1980, otras formas de agenciamiento que alteraron la normalidad de la vida pública impuesta por el terror dictatorial (Amigo et al., 2012). Los artistas se apropiaron de la noción misma de museo y de su capital simbólico, útil para establecer nuevos modelos institucionales desde el arte mediante la alteración de los postulados elitistas y coloniales de estructuras museales hegemónicas predominantes. Esta iniciativa, al margen o en contra del mercado, imprimió un giro en la manera de entender al público como sujeto activo en relación con el objeto artístico; de este modo la exposición pasó de considerarse como solo una exhibición de objetos, para ser pensada como un medio de comunicación. La forma invectiva en la que operan los artistas desfigura la manera convencional de relacionarse con el público, crea así ideas de exhibición basadas en la necesidad de hallar, en ese vínculo artista - público, determinadas formas de reciprocidad no estandarizadas que a su vez, recrearon las deficiencias institucionales.

Gustavo Buntinx, historiador de arte, crítico y curador oriundo de Buenos Aires, residente en Lima y autor de numerosos trabajos sobre artes visuales latinoamericanas, creó en 1983 Micromuseo ("al fondo hay sitio"), institución errante con una colección de objetos pensada para promover mediante la circulación y no la acumulación señalando esto último como función de los museos oficiales de la ciudad. En Latinoamérica, "micro" se refiere a lo pequeño, pero también se le llama así al autobús. Buntinx articuló el proyecto bajo la terminología que utilizan los choferes de los buses, empleó la cotidianeidad como estratégia e invitó a las personas a subirse y procurarse un lugar. Buntinx también expresó que no era director, ni curador del Micromuseo, sino, el conductor de un vehículo que transitaba por una gran autopista cultural y que además contaba con un "Taller de Mecánica" accesible e integrado por cinco personas, incluido él.

El Micromuseose planteó como una institución móvil, con unas estrategias expositivas ambulantes, que, concentró la mirada sobre los elementos de identificación cultural andinos con respecto al arte hegemónico, de esta manera proclamó sentido a las prácticas de arte local, soportadas en nociones como vacío museal y museotopía, encarando los museos de arte en la ciudad de Lima, ausentes e incapaces de cubrir las necesidades socioculturales emergentes del país, situación que convirtió en oportunidad para crear y debatir por medio del arte, nuevas estructuras expositivas.

Otra propuesta que también confronta la idea dominante del arte en relación con los circuitos oficiales por medio de una práctica colectiva es el Museo Bailable de Buenos Aires 1988, creado, curado y definido por Fernando "Coco" Bedoya que manifiesta: 
[...] Yo organizaba unos eventos llamados Museos bailables, que iniciaban como exposiciones y terminaban como recitales. Y toda la modificación se daba a través de la luz nada más. Empezaban como una exposición tradicional, de dos o tres artistas, colectiva, donde las luces se empezaban a apagar y había un ambiente teatral, pero no escenográfico, sino teatral de performance, de acción de cuerpo sobre el espacio, donde lo que sucedía en realidad, era la intensidad, lo que más se buscaba era el rozamiento. Eran como las exposiciones de Parafernalia, donde la intensidad pasa por el rozamiento. Entonces, yo trabajaba ese rozamiento cargándolo con una cantidad de actividades artísticas sobre ese mismo espacio, simultáneo. (2014, p. 106)

Más allá de proponer obras, "Coco" Bedoya, plantea apropiaciones urbanas y territoriales que convirtieron la práctica artística en un laboratorio de experiencias, que interceptó zonas comunes en las que se conjugaron lo erudito y lo artesanal, donde se agruparon diversas generaciones de artistas y convergieron distintas disciplinas que activaron la escena artística y transformaron la manera de experimentar el arte para aquellos que siempre estuvieron al margen.

Los anteriores ejemplos se formulan como espacios expositivos alternos o lugares extrainstitucionales que intentan subvertir el orden de lo establecido, como estrategias de prácticas artísticas con estructura desafiante, crítica, temporal y nómada. Una actitud que tiene filiación inmediata con Marcel Broodthaers y el Musée d'Art Moderne, Departement des Aigles, creado entre 1968-1972, Daniel Buren o Hans Haacke, artistas norteamericanos de los años sesenta,que cuestionaron su papel en la sociedad e hicieron una crítica a los soportes tradicionales de la pintura y la escultura. Todos, sin importar su origen o ubicación geográfica, inquirieron sobre sus límites y las maneras de presentar la obra de arte, asumieron distintos roles: coleccionistas, conservadores, curadores, críticos de arte, expandieron labores interdisciplinares que les permitieron multiplicar sus intenciones de ocupar un lugar sustancial en la esfera social y entablar una relación directa con los diversos públicos.

\section{Experiencias artísticas desde las artes plásticas en colombia}

La historia del arte se clasifica por período, escuela, estilo, movimiento o artista, lo cual impone a los objetos significados que se legitiman por medio de las instituciones culturales oficiales. De ahí que "[...] La presencia de una obra de arte en un museo (al menos, en las principales instituciones tales como la National Gallery de Londres o el Museo de Arte Metropolitano de Nueva York), ya representan en sí una forma de clasificación" (Barker, 1999, p. 13). En consecuencia, no es lo mismo si la obra está exhibida en el MoMA o en un barrio al norte de la ciudad de Bogotá, su singularidad y certificación depende del lugar en el que se alberga.

La Bienal de Venecia en Bogotá es una plataforma artística que promueve procesos creativos, la lideran estudiantes de la Universidad Nacional de Colombia, quienes decidieron organizar en 1995 una exhibición de arte en el barrio Venecia, "con la intención de desplazar el arte contemporáneo de sus habituales espacios, a otros no tan centralizados." (Aguirre, 2003). Esta movilidad en la que incurre el colectivo Matracas -y señala el director Franklin Aguirre- urge en el ambiente cultural capitalino para brindar nuevas trayectorias a las propuestas plásticas para generar otras formas de producción, circulación y apropiación de los artistas y artesanos locales desde lugares anónimos. Esta iniciativa genera gran impacto a nivel local y visibilidad en el ámbito cultural nacional, no solo porque las obras en la Bienal se crean a partir del vínculo que los artistas pueden establecer con la población y el barrio, también porque potencia "la idea de la obra 
de arte como herramienta para el cambio social" (Palacios, 2009, p. 199).

En esta experiencia artística y expositiva la metodología empleada de intervención-acción es cercana a la metodología etnográfica, ya que los artistas realizan trabajo de campo y basan todo el proceso de creación, desarrollo del evento y demás, en las dinámicas comunitarias. Para Aguirre (2003) esto implica:

[...] apoyar la búsqueda de la propia comunidad para transformar sus formas de vida, explorando con ella nuevos elementos de interpretación de su cotidianeidad, nuevos referentes para leer y experimentar su relación con el espacio urbano, el barrio y consigo mismos. (p. 129)

La Bienal toma el arte de incorporar la cultura a la cotidianeidad, abre el camino para proyectos que priorizan los beneficios comunes y sociales por encima del carácter estético de la obra. Esto significa abordar problemáticas ciudadanas, contextuales urbanas y culturales que hacen del objeto artístico, la gestión, organización comunitaria y técnicas de montaje, un dispositivo para indagar acerca de la memoria, las historias locales y la importancia de la participación colectiva en la construcción de un interés común en el seno de la esfera pública.

Por consiguiente, el intercambio entre el artista y la comunidad se convierte en acción fundamental para que instituciones como el Ministerio de Cultura de Colombia generen iniciativas que permitan ampliar la noción de prácticas artísticas más allá de una estética relacional (Bourriaud, 2009) enfocada en el vínculo íntimo que genera el artista con otros en circuitos artísticos tradicionales como museos y galerías o arte comunitario (Morgan, 1995), en el que el artista acompaña a la comunidad para recuperar zona marginadas o en conflicto por medio de bailes, murales, espacios arquitectónicos o actividades artísticas. "Son los laboratorios de creación en contextos regionales generados por el Área de Artes Visuales del Ministerio de Cultura desde 2004" (Gil, 2013, p. 171). Con estos, el Estado colombiano pretende democratizar la cultura y fortalecer prácticas artísticas que abordan el ejercicio curatorial, museológico, proyectos de intervenciones creativas rurales y urbanas, propuestas de conservación de prácticas y saberes de culturas indígenas, indagaciones del arte relacionada con la pedagogía entre otras líneas de experimentación, para enriquecer el lenguaje artístico y la experiencia creadora.

Este emprendimiento, además de atender las regiones, apunta a formar públicos culturales en un Estado que aún no advierte que aprender a valorar el arte es parte fundamental del crecimiento y desarrollo de todas y cada una de las personas que integran el país. Es así que, la formación se entiende desde la aplicación del currículo en la educación básica primaria o de pequeñas muestras y representaciones limitadas de la cultura y los territorios de pueblos indígenas y afrocolombianos. Si bien es cierto que estas iniciativas son importantes, no es menos cierto que cuando la cultura se considera presente solo en el marco de reinados y festivales, las voces e imaginarios colectivos se uniforman y debilitan. Por ende, la siguiente cita de Néstor García Canclini (2008): “El Estado, no crea cultura. Es indispensable para generar las condiciones contextuales, las políticas de estímulo y regulación en las cuales puedan producirse bienes culturales y se puedan acceder a ellos con menores discriminaciones". (p. 71)

A partir de esta premisa, la articulación de proyectos nacionales que invitan al diálogo y la participación no son del todo efectivos por las condiciones desiguales de desarrollo en que se encuentran las regiones y porque muchas veces, los procesos creativos que allí se originan no culminan en productos tangibles o comercializables. Sin embargo, es importante destacar el riesgo que asumen todos los agentes en la idea de construir espacios culturales 
para crear memoria por medio de los diversos proyectos que articulan distintos lenguajes artísticos, en función de generar redes asociadas a circuitos académicos o comunicacionales para movilizar y visibilizar estas prácticas inscritas en la pedagogía, la gestión cultural y el arte.

Es precisamente en este escenario en el que se desarrolla la primera fase del proyecto Prácticas y saberes comunitarios para la creación artística. "Aportes a la construcción de paz en el suroccidente colombiano". El objetivo es construir procesos creativos junto al colectivo Ashampa Awáz como alternativa para fortalecer el pensamiento propio y las identidades de las comunidades que siempre fueron negadas, rechazadas y empobrecidas fuera de su territorio. De igual forma, busca alentar las acciones participativas basadas en manifestaciones estéticas, que si bien no apuntan a crear obras de arte, reivindican saberes como el tejido de la igra3 por medio de experiencias creativas plásticas, acontecimientos y reflexiones desde los territorios. Es así como la función tradicional del artista-investigador que toma lo que necesita y lo exhibe con su firma se desdibuja y los circuitos artísticos o académicos son solo una ruta posible para validar la intuición y la imaginación en diálogo con otras prácticas y saberes. Por tanto, el proceso creativo es, como bien lo mencionó Camnitzer, "[...] el acto cultural transformativo y de adquisición de conocimientos que se produce a través del ejercicio artístico que no necesariamente produce obra" (Revuelta, 2017).

En virtud de ello, las prácticas artísticas orientadas a las artes plásticas y visuales mencionadas en el texto señalan que la relación entre artista y comunidad va más allá de la creación de obras. Con ello, se pretende hacer hincapié en el proceso de creación como un espacio que genera dinámicas que propician una forma de conocer y apropiarse del mundo diferente a la establecida por las metodologías del razonamiento científico. De manera que esta forma singular (más que un método) sirva para acompañar los esfuerzos de este colectivo para preservar sus tradiciones y permanecer en el territorio desde otro lugar, pues, "la calidad de vida y el territorio no son las únicas pérdidas que amenazan a pueblos indígenas colombianos como los awá. La lengua, las tradiciones y las costumbres tienden a desaparecer con el desplazamiento" (Acnur, 2018).

\section{Conclusiones}

En primer lugar, analizar los actos creativos requiere un ejercicio de reconstrucción, que, sin duda, está atravesado por el incremento de estructuras económicas extravagantes que redefinen la creación artística y su ejecución. No obstante, el ejercicio creativo desborda de manera continua estos límites, ya que los artistas descolocan su rol protagónico para fungir como mediadores de acciones que pueden llegar a cuestionar las funciones de los espacios políticos imperantes, se superan así las instancias del arte incorporado al mercado, al conseguir renovar asiduamente su interrelación con la comunidad, muchas veces sin necesidad del objeto artístico.

Como segunda medida, cuando un artista pretende desarrollar sus iniciativas en una comunidad organizada, debe seguir los lineamientos que ésta exige para la aprobación, coordinación, seguimiento y cumplimiento del proyecto. Por supuesto, no hay una receta para conseguir integrarse a un contexto colmado de particularidades, lo que sí es evidente es la importancia de este acercamiento horizontal que suscita un sitio de intercambio, provocación, creación, voz y significado. Así, el proceso creativo ubica a los objetos y sujetos en una trama territorial que define las estrategias de comunicación,

2 Según la Organización Nacional Indígena de Colombia (ONIC), en la lengua indígena Awapít, que pertenece a la familia lingüística chibcha, "awá" significa la gente de la montaña, la gente de la selva" (ONIC, s. f.)

3 La igra Tejido tradicional de la Comunidad Awá, en los que representan relatos de su cosmogonía y significados de la lengua hablada por esta comunidad ancestral. 
redistribuye el flujo de información de la experiencia artística y destaca su lugar de emplazamiento.

El proyecto de investigación PySCCA Prácticas y saberes comunitarios para la creación artística. "Aportes a la construcción de paz en el suroccidente colombiano" localiza en el lenguaje visual ancestral (el tejido de la igra) y el contemporáneo occidental (prácticas artísticas) un punto de encuentro que funciona como vehículo de significados y valores, se aventura a la rehabilitación de la memoria y la historia étnica como parte vital de la identidad personal que, aunque puede o no aportar a la construcción Paz, sí, pretende crear modos de comunicar los tiempos-territorios que se habitan.

En consecuencia, el papel protagónico que cumple artista-comunidad es indivisible, aunque sus perspectivas pueden no ser necesariamente convergentes o compatibles entre sí, ambos experimentan los efectos del mundo globalizado, en el que lo cultural enlazado a lo económico bosqueja un nuevo orden simbólico, en el que lo social y lo comercial se cruzan; en el que la apatía y la celeridad se amplifican, incluso en los ejercicios socioculturales cuya fuerza simbólica aumenta, al mismo tiempo que examina lógicas de funcionamiento que responden a las necesidades actuales de comunicación. Estos aspectos discordantes incitan la creación de espacios en los cuales se confrontan nociones estandarizadas de experiencias sensibles que, a su vez, deben encontrar cómplices que desplieguen estrategias de producción, distribución y consumo, callejeras, en línea, errantes, nómadas, formas posibles de construir comunidad mediante procesos artísticos que logren intervenir en la industrialización de la cultura y permanecer al margen. Quizá esta coalición disfrazada de alianza provoque un instante democratizado e instituyente.

\section{Referencias}

Acha, J. (1979). Arte y sociedad: Latinoamérica, el sistema de producción. Fondo de Económica.
Aguirre, Franklin (2003). La Bienal de Venecia de Bogotá. Rodríguez, V. M. (ed.) (pp. 129136, 2003). Prácticas artísticas, Enfoques Contemporáneos. Bogotá, Universidad Nacional de Colombia e Instituto Distrital de Cultura y Turismo.

Amigo, R., Badawi, H., Biczel, D., Carvajal, F., Colombino, L., Cristi, N., Davis, F., Expósito, M., Gamarnik, C., García F., García Pérez de Arce, I., Gutiérrez Castañeda, D., Henaro, S., Keller, A., La Rocca, M., Longoni, A., López, M. A., Lucena, D., Manzi, J., ... Weiss, R. (2012). Perder la forma humana. Una imagen sísmica de los años ochenta en América Latina (catálogo). Museo Nacional Centro de Arte Reina Sofía. https://www. museoreinasofia.es/publicaciones/perder-forma-humana-imagen-sistimica-anos-ochenta-america-latina

Báez, E. (2000). Otto Dix: serie gráfica sobre la guerra. Revista Anales del Instituto de Investigaciones Estéticas, 22(76), 237-251. http://www.scielo. org.mx/pdf/aiie/v22n76/v22n76a7.pdf

Barker, E. (ed.) (1999). Contemporary Cultures of Display. Art and it's Histories. Yale University Press y Open University. https://gallowayexploringart.files.wordpress.com/2014/08/barker_intro_cont-cult-display.pdf

Bourriaud, N. (2009). Radicante. Adriana Hidalgo Editora.

Buntinx, G. (s. f.). Qué es y qué quiere ser Micromuseo ("Al fondo hay sitio"). Manifiesto de viaje. https://micromuseo.org.pe/manifiesto/index.html

Fundación Mapfree. (2010, enero 13). Impresionismo, Un Nuevo Renacimiento. Dossier de prensa. Recuperado de https://wwu.fundacionmapfre.org/fundacion/es_es/images/dossier-prensa-impresionismo-es_tcm1069-553479.pdf 
García, Canclini N, (2008). Latinoamericanos buscando lugar en este siglo. Paidós.

Gil, J. (2013). Los Laboratorios de Creación en contextos regionales. En Segundo encuentro de investigaciones emergentes ( $\rho p$. 171-186). Universidad Jorge Tadeo Lozano.

Giünta, A. (2014). ¿Cuándo empieza el arte contemporáneo? Fundación ArteBA.

Guerrero, C. (2016, junio 27). La Bienal de Venecia de Bogotá, es mayor de edad. Sus primeros 21 años. Fashión Radical Magazine. http://wuww. fashionradicals.com/la-bienal-de-venecia-debogota-es-mayoria-de-edad-sus-primeros21-anos/

Guilbaut, S. (2007). De cómo Nueva York robó la idea de arte moderno. Editorial Tiran lo Blanch.

Huici, F. (1981, agosto 19). Alfred H. Barr y la noción contemporánea del museo. El País. https://elpais.com/diario/1981/08/19/cultura/367020009_850215.html

Mendogo Minsongui, D. (2004). Los nuevos soportes de la creación artística: La creación artística africana, de la oralidad a la modernidad. Artes y Nuevas tecnologías: x Congreso de la Asociación Española de Semiótica (pp. 803-813). https://dialnet.unirioja. es/servlet/articulo?codigo=940488

Morgan, S. (1995): "Looking back over 25 years". En Dickson, M. (ed.)

Art with People. Sunderland. AN Publications.

Ordoñez, J., y Vergara, M. (1999). Páginas sobre la expresión artística. Revista Arte, individuo y sociedad, (11) 39-46. https://revistas.ucm.es/index. php/ARIS/article/view/ARIS9999110039A/5944
Organización Nacional Indígena de Colombia (ONIC). (s. f.). Awa. https://wwww.onic.org.co/ pueblos/112-awa

Palacios Garrido, A. (2009). El arte comunitario: origen y evolución de las prácticas artísticas colaborativas. Revista Arteterapia, 4, 197-211. https://revistas.ucm.es/index.php/ARTE/article/ view/ARTE0909110197A

Read, H. (1954). El significado del arte. Losada Editor.

Revuelta, L. (2017, febrero 2). Entrevista. Camnitzer Luis: "el arte es un instrumento para mejorar la sociedad". ABC cultural. https://www.abc.es/cultura/cultural/abci-arte-instrumento-para-mejorar-sociedad-201702020153_noticia.html

Schwitters, K. (1995). Merz. Catálogo exposición 6 abril-18 junio 1995 (рp. 184-187). IVAM Centre Julio González. https://merzmail.files.wordpress.com/2015/03/kurt_schwitters_merzmail_net.pdf

Quijano, Rodrigo (2014). Fernando "Coco" Bedoya. Mitos, acciones e lluminaciones. Malí, Museo de Arte de Lima. Perú.

Thomas, E. (2012). En busca del arte perdido: Merzbaue de Kurt Schwitters. Insade/out MoMA Recuperado de: :https://wuw.moma. org/explore/inside_out/2012/07/09/in-searchof-lost-art-kurt-schwitterss-merzbau/

UNHCR ACUNR (2018) Indígenas Colombianos amenazados por el conflicto en Colombia. Comité Español de ACNUR. Recuperado de: https://eacnur.org/es/actualidad/noticias/historias-de-vida/indigenas-colombianos-amenazados-por-el-conflicto-en-colombia\#: :text=La\%20 calidad\%20de\%20vida\%20 y,nativa\%20fuera\%20de\%20su\%20territorio. 